\title{
Spontaneous Uterine Perforation in a Choriocarcinoma Presenting with Pyoperitoneum
}

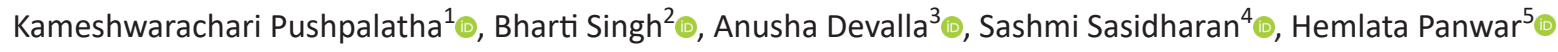

\begin{abstract}
Aim and objective: Choriocarcinoma can have intricate presentations and pose challenges in management. We are presenting a rare case of spontaneous uterine perforation in a choriocarcinoma.

Background: Choriocarcinoma is a rare neoplasm with varied incidence worldwide. It is a highly malignant tumor of trophoblastic tissue.

Case description: We present a case of 29-year-old multiparous women admitted with fever and foul-smelling vaginal discharge. After 2 days of admission, she developed features of perforation peritonitis. She was taken up for emergency laparotomy. Hysterectomy was performed in view of perforation of uterus with extensive necrosis. Peritoneal cavity was filled with pus. The histopathological examination revealed choriocarcinoma. Her serum beta-human chorionic gonadotropin (hCG) was $2397 \mathrm{mIU} / \mathrm{mL}$. She received multiagent chemotherapy (EMA/CO) for six cycles till the beta-hCG values came to normal. On 1 year follow-up, the beta-hCG values remained normal.

Conclusion: Choriocarcinoma presenting with acute abdomen and pyoperitoneum is a rare clinical presentation and should be a kept as a diagnosis of exclusion.

Clinical significance: Timely diagnosis and management can improve the clinical prognosis of the patients.

Keywords: Acute abdomen, Choriocarcinoma, Laparotomy, Pyoperitoneum, Uterine perforation.

Journal of South Asian Federation of Obstetrics and Gynaecology (2021): 10.5005/jp-journals-10006-1946
\end{abstract}

\section{BACKGROUND}

Most cases of choriocarcinoma are intrauterine and are of gestational origin. ' Extrauterine gestational choriocarcinomas may also arise at the site of ectopic pregnancy. ${ }^{1}$ The nongestational choriocarcinomas are believed to develop from pluripotent germ cells, most commonly arising in the gonads. ${ }^{1}$ Gestational choriocarcinoma is a rare complication of pregnancy and usually arises from a prior molar pregnancy or rarely a nonmolar gestation, within 1 year of the antecedent pregnancy. ${ }^{2}$ It usually presents with amenorrhea and irregular bleeding. It can present any time after the termination of primary pregnancy, but patients who present early have a better prognosis. The rarity in our case was that the patient developed pyoperitoneum and sepsis due to perforation of choriocarcinoma, which is extremely rare.

\section{Case Description}

A 29-year-old multiparous woman was admitted with complaints of foul-smelling vaginal discharge and fever for 2 months. She gave a history of evacuation 4 months back at a local hospital. The evacuation was done for history of 2 months amenorrhea. She was P3L3A1 and the last childbirth was 5 years back. On examination, the patient was severely pale with poor general condition. Per abdomen, the uterine size was corresponding to 12-14 weeks gestation, and globular and bilateral adnexal palpation was unremarkable. Ultrasound was done which revealed an enlarged uterus with a heterogeneous lesion arising from the body and fundus of the uterus.

Magnetic resonance imaging (MRI) pelvis revealed a uterus measuring $11.5 \times 8.9 \times 15.3 \mathrm{~cm}$ with loss of endomyometrial differentiation and a large ill-defined heterogeneous lesion with serosal and parametrial extensions suggesting gestational trophoblastic neoplasia, most likely invasive mole/choriocarcinoma. Both ovaries showed $3.5 \times 2.3 \mathrm{~cm}$ unilocular nonenhancing cystic
${ }^{1-4}$ Department of Obstetrics and Gynecology, All India Institute of Medical Sciences, Bhopal, Madhya Pradesh, India

${ }^{5}$ Department of Pathology, All India Institute of Medical Sciences, Bhopal, Madhya Pradesh, India

Corresponding Author: Bharti Singh, Department of Obstetrics and Gynecology, All India Institute of Medical Sciences, Bhopal, Madhya Pradesh, India, Phone: +91 9827008308, e-mail: drbharti01@ gmail.com

How to cite this article: Pushpalatha K, Singh B, Devalla A, et al. Spontaneous Uterine Perforation in a Choriocarcinoma Presenting with Pyoperitoneum. J South Asian Feder Obst Gynae 2021;13(4):272-274.

Source of support: Nil

Conflict of interest: None

lesions. Within 2 days, the patient developed features of acute abdomen. Examination revealed a tense, tender, and rigid abdomen for which patient underwent an emergency laparotomy. Peritoneal cavity was filled with purulent fluid, uterus was $14 \times 10 \times 8 \mathrm{~cm}$ size with a $2 \times 2 \mathrm{~cm}$ perforation in the left posterior uterine wall, and pus was oozing from it (Fig. 1A). Bilateral tubes and ovaries were grossly normal. On cut section of the uterus, endometrial cavity was dilated and filled with friable grey white to grey brown mass. Pus was seen in the cavity and necrosis was extending till the serosa in the posterior wall (Fig. 1B). Hysterectomy with bilateral salpingectomy and thorough peritoneal lavage was performed. Intraoperatively, the patient received 2-unit packed red blood cell (PRBC) and 1 was transfused postoperatively. Serum beta-hCG value was $2397 \mathrm{mlU} / \mathrm{mL}$.

Histopathological examination of the specimen revealed choriocarcinoma invading the myometrium and the serosa was also invaded posteriorly (Fig. 2). Bilateral tubal histology 

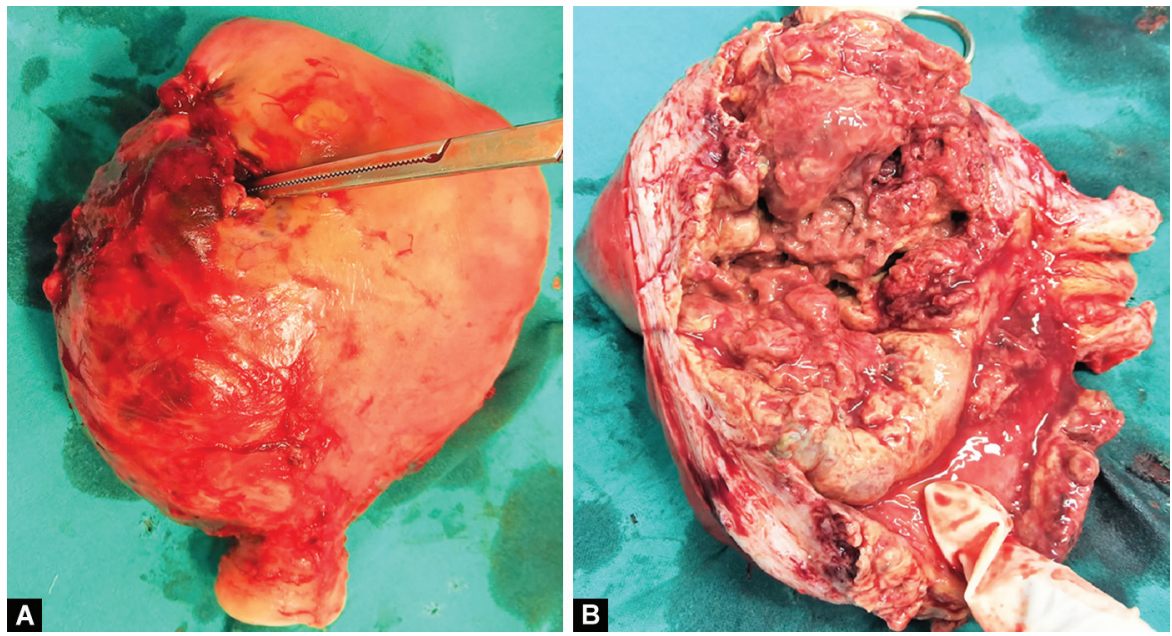

Figs $1 \mathrm{~A}$ and $\mathrm{B}$ : (A) Posterior surface of the uterus showing perforation; (B) Cut section showing necrosis and pus inside the cavity
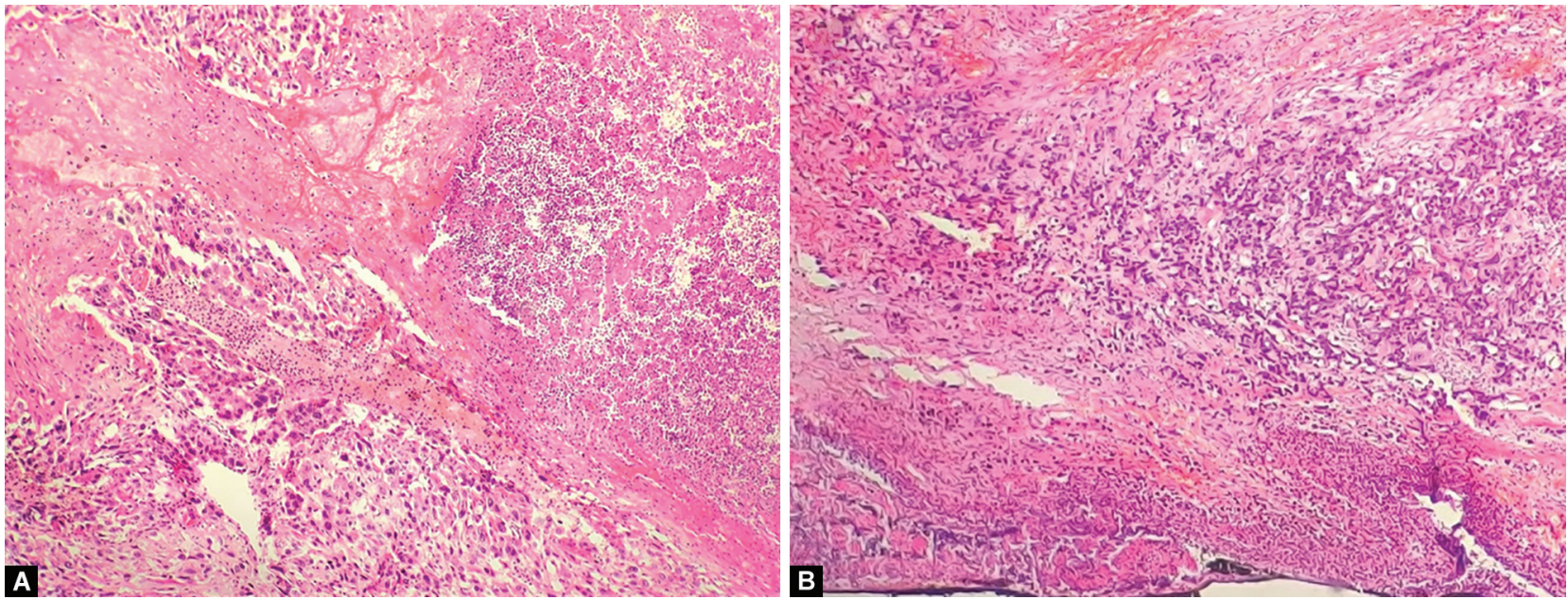

Figs $2 A$ and B: (A) H\&E section showing cytotrophoblast and syncytiotrophoblast with intervening areas of fibrinoid necrosis, inflammation, and hemorrhage; (B) Posterior wall of uterus showing tumor till serosa (40X)

was unremarkable. Fluid/pus cytology report was suggestive of predominantly inflammatory infiltrates of neutrophils, few macrophages, and scattered reactive mesothelial cells. No atypical or malignant cells were seen. Postoperatively, MRI brain was normal. Contrast-enhanced computed tomography (CECT) chest had multiple small soft tissue density nodules scattered diffusely in bilateral lung parenchyma suggestive of metastatic deposits. The final diagnosis was choriocarcinoma stage-III. Initially, single agent chemotherapy (methotrexate) was administered to the patient for two cycles with alternate oral rescue doses of folinic acid. In view of unsatisfactory response, EMA/CO regimen (etoposide, methotrexate, actinomycin D, cyclophosphamide, and vincristine) was initiated. This was accompanied by strict daily clinical evaluation, regular hemogram, liver and renal function tests, and beta-hCG follow-ups after every cycle. The patient had received injection filgrastim intermittently for chemotherapyinduced neutropenia. Chemotherapy was continued for six cycles until beta-hCG came to normal. Patient was reassessed after 1 year. There were no fresh symptoms and serum beta-hCG was normal.

\section{Discussion}

The clinical presentation of choriocarcinoma can be varied. Hemoperitoneum secondary to invasive mole is a common presentation but is rarely seen in choriocarcinoma. ${ }^{3}$ The exact pathogenesis of rupture of the uterus in choriocarcinoma is not known. However, various theories have been proposed to explain the mechanism of uterine rupture. ${ }^{4}$ Malignant trophoblasts invade the uterine veins and damage the blood vessels. ${ }^{4}$ Subsequent to the vascular damage, multiple infarctions do occur due to thrombosis, vascular aneurysms, and intratumoral bleeding. ${ }^{4}$ It should be typically differentiated with other causes of acute abdomen. Patients of choriocarcinoma presenting with acute abdomen due to pyoperitoneum or hemoperitoneum caused by perforation of uterus usually require hysterectomy as a lifesaving procedure.

The International Federation of Obstetrics and Gynaecology (FIGO) grading and scoring system is recommended for grading the disease into the low-risk and high-risk groups. As these tumors 
are highly sensitive to chemotherapy, single-agent and multiagent chemotherapies are advised according to the risk scoring.

\section{Conclusion}

Our case emphasizes the role of surgical intervention at proper time along with the chemotherapy. Early recognition and initiation of chemotherapy is a well-known determinant of prognosis of choriocarcinoma.

\section{Clinical Significance}

Recognition of variation of presentation of choriocarcinoma and timely management is key to the successful treatment.

\section{ORCID}

Kameshwarachari Pushpalatha (1) https://orcid.org/0000-00029640-6026

Bharti Singh 10 https://orcid.org/0000-0003-2848-760X
Anusha Devalla @ https://orcid.org/0000-0002-4744-8799

Sashmi Sasidharan (1) https://orcid.org/0000-0003-3868-4666

Hemlata Panwar (1) https://orcid.org/0000-0001-7591-282X

\section{REFERENCES}

1. Fisher RA, Savage PM, MacDermott C, et al. The impact of molecular genetic diagnosis on the management of women with hcg-producing malignancies. Gynecol Oncol 2007;107(3):413-419. DOI: 10.1016/ j.ygyno.2007.07.081.

2. O'Neill CJ, Houghton F, Clarke J, et al. Uterine gestational choriocarcinoma developing after a long latent period in a postmenopausal woman: the value of DNA polymorphism studies. Int J Surg Pathol 2008;16(2):226-229. DOI: 10.1177/1066896907307038.

3. Liberis V, Bouchlariotou S, Ammari A, et al. Acute abdomen as initial presentation of gestational choriocarcinoma. Arch Gynecol Obstet 2009;280(5):859-862. DOI: 10.1007/s00404-009-0968-5.

4. Ma Y, Xiang $Y$, Wan $X R$, et al. The prognostic analysis of 123 postpartum choriocarcinoma cases. Int J Gynecol Cancer 2008;18(5):1097-1101. DOI: 10.1111/j.1525-1438.2007.01132.x. 